\title{
Causes of Rheumatism
}

\author{
Zhe Yin \\ Department of Mathematics, Yanbian University, China
}

\begin{abstract}
This article clarifies the causes of rheumatism.
\end{abstract}
Keywords: rheumatism; light; gravitational wave

\section{INTRODUCTION}

It has been proposed in 2010 that substances change to the direction of increase of atomic weight and molecular weight (Zhe Yin , 2010) and it has also been proposed that spiral trajectory of gravitational waves (Weber, J., 1959 and 2016) and the variable force field is the condition of energy conversion in February 2016 (Zhe Yin and Liu, 2016a,b). The motion trajectory of the electrons, proposed the law of motion that, spiral trajectory gravitational wave produces entanglement in the process of convergence and divergence, increases the energy, in the trajectory of Mobius', and spiral gravitational wave convergences inwards in March 2016 (Zhe Yin, 2016; Zhe Yin, Liu, 2016a,b). Inward convergence is equivalent that the centripetal force is greater than buoyancy and other external field forces, outward divergence equivalent buoyancy and other external field force is greater than the centripetal force. The following description provides the use of gravitational wave theory, to elucidate the propagation pattern of light between the electrons of the gravitational waves. The light has the entanglement between the periodic and the optical blocks with the same properties. The degree of entanglement between the light blocks is expressed by relative energy. Each photoperiod contains four sub-process morphologies, each sub-process morphology, forming a separate, electron-group and entangled form of relative energy set. This article uses the principle of light to clarify the causes of rheumatism.

\section{THEORETICAL PREPARATION}

Theorem 1 (Gravitational waves existence theory) (Zhe Yin et al., 2016; Zhe Yin, 2017a, b, c,d,e,f).

There are A, B two points. A is the wave source of gravitational field, and B is a point in the gravitational field. The existence of energy rotational motion (including proton, neutron, atomic nucleus or planet) at $\mathrm{A}$ is the necessary and sufficient condition of the existence of gravitational waves at $\mathrm{B}$. Direction is the bidirection of the path tangent at point $\mathrm{B}$, and the limit of the convergence direction is a point.

Theorem 2 (Gravitational wave stability theory) (Zhe Yin et al., 2016; Zhe Yin, 2017a, b,c,d,e,f)

A is a gravitational wave source, $\mathrm{B}$ is a point in gravitational field of A. The necessary and sufficient conditions of stable gravitational wave at B point are:

$$
\frac{d r}{d \theta}=b \quad \text {, (b is a constant). }
$$

Inference of theorem 2: If a gravitational wave track meet Archimedean spiral, this must be stable gravitational waves. 
There is, $r=a+b \theta$.

$\mathrm{r}$ is the distance of $\mathrm{A}$ to $\mathrm{B}$; $\mathrm{a}$ is the spiral length of $\mathrm{A}$ to $\mathrm{B} ; \mathrm{b}$ is the distance between the pirals.

\section{THE NATURE OF LIGHT AND THE CAUSES OF RHEUMATISM}

According to the gravitational wave equation, the periodic law of light is obtained. gravitational waves are the propagation of light. Light is the same form of electronic, ntangled particles in the form of transmission. Photons have relative energy and quality. Through the electronic entanglement, supplement and increase the energy, gradually increase the ave length.

$r=a+b \theta \quad \theta_{\text {is the radian, the cycle is } 2 \pi}$.

Theorem 1: The wave of light is periodic and its period is $2 \pi$.

According to the independence, symmetry, monotonic principle, light has only four forms. Four sub-process morphologies of the photoperiod).

\section{Theorem 2: Four independent morphological equations of light}

The first sub-process morph equation: $r=a+b(2 k \pi+0), k=0,1,2,3, \ldots$.

The second sub-process morph equation: $r=a+b\left(2 k \pi+\frac{\pi}{2}\right), k=0,1,2,3, \ldots$

The third sub-process morph equation: $r=a+b(2 k \pi+\pi), k=0,1,2,3, \ldots$.

The fourth sub-process morph equation: $r=a+b\left(2 k \pi+\frac{3 \pi}{2}\right), k=0,1,2,3, \ldots$

As in different environments, hydrogen atoms can be positively charged or negatively charged, the electron entangled state (photon) can be positively charged or negatively charged. Each sub-process of electrons forms a separate set of photonics form and share relative energy.

Anywhere on earth, four kinds of photon clusters coexist. The relative energy of the photon is proportional to the wavelength.

The four bases of DNA receive energy, and the two deoxynucleotide chains store energy. The energy is used for DNA transcription. When the genes are produced, DNA receives four photon sets of energy. The first photon subset and the fourth photon subset are monotonically increasing in energy. When the DNA metabolic energy is stored, only the first photon and the fourth photon are collected.

Rheumatosis Pathogenesis: DNA base A and T, G and C accept energy, generate electrical energy, stored in two spiral chains. DNA energy is used for transcription. Among them, DNA acid energy comes from light energy. DNA alkaline energy from the food. DNA base between the hydrogen, plays the role of the valve. Human body to accept the lack of light energy, is the first cause of rheumatism. Base and two deoxynucleotides between the potential difference is not large enough, leading to metabolic transport is not smooth, generate a lot of small molecule mRNA and small molecule miRNA. Accelerating the propagation of biological channels of the entrance cells and the proliferation of connective tissue cells, resulting in smaller diameter of the organ channel. Water molecules are deposited, are not smooth, and the hydrogen bases between the DNA bases are destroyed, and the potential difference is permanently destroyed (not full of electricity or leakage). This is the second cause of rheumatism. 
Valve function of the hydrogen, the greater the potential difference between the two bases, the greater the potential difference, the better the insulation function. Figure 1.

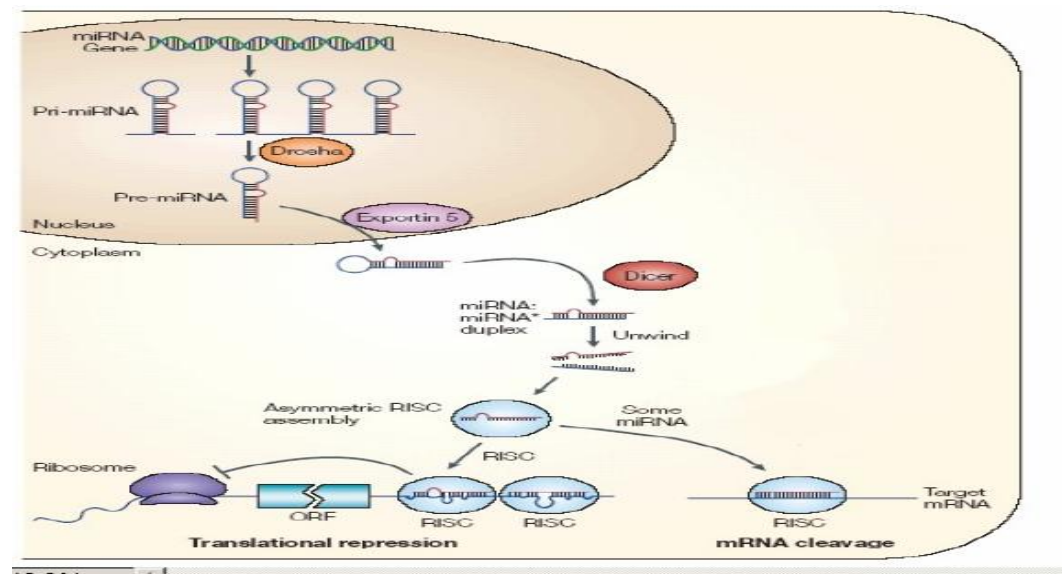

Figure1.

According to the literature (Zhe Yin et al., 2012,2014,2015; Zhe Yin, 2017d,e,f), connective tissue cell proliferation is 3 times the muscle cell reproduction. Connective tissue cell channel minimum, is also a necessary condition.

Treatment of rheumatism Note: can not rush to expand the diameter of the organ cell channel. Because the expansion of organ cell channels is the root cause of the onset of diabetes.

\section{Conclusion}

This paper uses the gravitational wave properties to elucidate the causes of rheumatism. The relevant research will play a due role.

\section{REFERENCES}

Weber, J., 1959, 2016. Gravitational Wave, Laser Interferometer Gravitational Wave Observatory. 2016.2.11.23, 30 .

Zhe yin, 2010. The co-planarity and symmetry principle of earthquake occurrence. Int. J. Geosci. 1(1), $38-43$.

Zhe yin, 2016. Zhe Yin's energy and wave theorem. Int. J. Engg. Adv. Res. Technol. 2(3), 44-45.

Zhe yin, 2017a. The function and relation of proton and neutron. Int. J. Recent Sci. Res. 8(1), 15321-15324.

Zhe yin, 2017b. Effect of Gravitational Wave on DNA Base. Int. J. Recent Sci. Res. 8(2), 15705-15707.

Zhe yin, 2017c. The formation of elements. IOSR J. Appl. Phys. 9(2), 08-13.

Zhe yin, Liu, D., 2016a. Electron trajectory. Int. J. Appl. Sci. Math. 3( 2), 56-57.

Zhe yin, Liu, D., 2016b. Causes of sound wave on the moon. Int. J. Eng. Appl. Sci. 3(2), 56-58.

Zhe yin, Zhang, W., Bie, H., 2016. Zhe Yin's theorem of molecular biology. Acad. J. Sci. Res. 4(4), 99-102.

Zhe yin, 2017d. The periodicity of light and the nature of chlorophyll. J. Curr. Res. Biosci. Plant Biol. 4(4), 46-48.

Zhe yin,yunfei Guo,2014. Application of the ZheYin's Gene Inherits Law, Open Journal of Genetics, Vol.4, No.6,pp:434-438.

Zhe yin, 2012. The Law of Genetic Inheritance, Open Journal of Genetics, Vol.2, No.1,pp: 47-50.

Zhe yin, 2017e. EFFECTS OF GRAVITATIONAL WAVE ON GENETIC INHERITANCE, European Journal of Basic and Applied Sciences, Vol. 4 No. 1, pp:35-40.

Zhe yin,2017f.Visible Light Formation Process, Journal of Applied Physics (IOSR-JAP), Volume 9, Issue 2, PP 37-39.

Zhe yin,yunfei guo,2015.Bio-segregation research to understand the biological metabolism, Pure Appl. Biol., 4(2): 259-260. 